\title{
Interfacing liquid chromatography with atmospheric pressure MALDI-
} MS

\section{Journal Article}

\section{Author(s):}

Daniel, Jürg M.; Laiko, Victor V.; Doroshenko, Vladimir M.; Zenobi, Renato

Publication date:

2005-11

Permanent link:

https://doi.org/10.3929/ethz-b-000030655

Rights / license:

In Copyright - Non-Commercial Use Permitted

Originally published in:

Analytical and Bioanalytical Chemistry 383(6), https://doi.org/10.1007/s00216-005-0098-2 
Jürg M. Daniel • Victor V. Laiko •

Vladimir M. Doroshenko · Renato Zenobi

\section{Interfacing liquid chromatography with atmospheric pressure MALDI-MS}

Received: 16 June 2005 / Revised: 18 August 2005 / Accepted: 31 August 2005 / Published online: 28 October 2005

C) Springer-Verlag 2005

\begin{abstract}
Two different strategies for coupling liquid chromatography with atmospheric pressure matrix assisted laser desorption/ionization (AP MALDI) are presented. The first method is flow-injection liquid AP UV-MALDI. Compared with previous similar research, the detection limit was improved 10 times to $8.3 \mathrm{fmol}$ using a solution of $50 \mathrm{nM}$ peptide with $25 \mathrm{mM} \alpha$-cyano-4-hydroxycinnamic acid. The applicability of this method to measure oligosaccharides, actinomycin antibiotics, antibiotics, phosphopeptides, and proteins is demonstrated. The upper mass limit achieved with the current instrumentation is 6,500 $\mathrm{Da}$ (doubly charged cytochrome $c$ ). The feasibility of a second strategy based on single-droplet IR AP MALDI is demonstrated here. Aqueous peptide solutions were successfully measured by this method.
\end{abstract}

Keywords Matrix assisted laser desorption/ionization · IR ionization from solution - IR matrix assisted laser desorption/ionization - Liquid matrix · Atmospheric pressure matrix assisted laser desorption/ionization

Matrix assisted laser desorption/ionization (MALDI) timeof-flight (TOF) mass spectrometry (MS) is, besides electrospray ionization (ESI), one of the most important analytical tools in the investigation of proteins, peptides, nucleic acids, organometallic compounds, and synthetic polymers. Since the development of the technique by Hillenkamp and Karas [1-3] as well as initial efforts by Tanaka [4], significant improvements of the method have

J. M. Daniel $\cdot$ R. Zenobi $(\bowtie)$

Department of Chemistry and Applied Biosciences,

Swiss Federal Institute of Technology (ETH),

Hönggerberg,

8093 Zürich, Switzerland

e-mail: zenobi@org.chem.ethz.ch

Fax: +41-1-6321292

V. V. Laiko · V. M. Doroshenko

MassTech Inc.6992 Columbia Gateway Drive,

Columbia, MD 21046, USA been achieved. A wide variety of different matrices have been discovered [5-11], and, in addition, matrix-free techniques, such as desorption/ionization on silicon [12, 13], have been developed. In 2000, Laiko et al. [14, 15] introduced atmospheric pressure (AP) MALDI . Desorption and ionization in AP MALDI take place at AP, outside the mass spectrometer. An AP interface is used to transfer the ions into the high vacuum of the mass analyzer. AP MALDI can be used as a quasicontinous ion source by employing high repetition rate lasers for the desorption. AP MALDI sources can be coupled to any mass spectrometer with an AP interface (ESI instruments), allowing the use of less expensive quadrupole ion traps (QIT) instead of TOF analyzers. Also, AP MALDI-MS offers the most cost effective solutions for tandem MS with ion trap spectrometers instead of MALDI-TOF/TOF or MALDI-QTOF instruments [16].

In addition to the easier sample introduction for AP MALDI there are some major advantages compared with conventional vacuum MALDI. AP MALDI is very tolerant to variations in laser fluence which is not influencing the resolution of the mass spectra obtained. Furthermore, ions with lower internal energies are produced, which is beneficial for the analysis of noncovalent complexes or fragile analytes [17], such as phosphorylated and glycosylated peptides. Unwanted clustering between matrix and analyte ions can be partially eliminated by increasing the laser energy or by tuning the AP interface parameters [14].

A key practical advantage of AP MALDI arises from the fact that the operation at AP completely eliminates the need for introducing a sample probe into the high vacuum of the mass spectrometer. This permits interfacing it on-line with liquid chromatography (LC) or capillary electrophoresis. Furthermore it allows the use of volatile matrices, in particular liquid matrices, such as water and codissolved matrices. Ring and Rudich [18] and Zöllner et al. [19] have shown that for vacuum MALDI liquid matrices provide excellent shot-to-shot reproducibility and long-term analyte signal stability. It was shown that water is well suited as a solvent and matrix for IR MALDI [10, 20, 21]. In previous work we demonstrated [22] the potential of 
different matrices codissolved in water or methanol for the analysis of small peptides and polymers by AP UVMALDI. We have also shown that quantitative measurements are achievable with these systems. These findings are supported by a recent publication of Turney and Harrison [23]. MALDI shows a higher tolerance to nonvolatile organic and inorganic compounds compared with ESI. Therefore LC-MALDI-MS is superior when nonvolatile buffers or trifluoroacetic acid are used in the mobile phase of the chromatography. Furthermore, on-line coupling of LC to AP MALDI delivers an immediate result of the separation and the analysis time is significantly reduced compared with that for off-line coupling methods.

Here we describe the specifications achieved for a flowinjection AP UV-MALDI source and its potential to be used as a detector for LC experiments. We demonstrate the successful measurements of different classes of compounds varying from oligosaccharides, actinomycin antibiotics, phosphopeptides to small proteins. The upper mass limit as well as the limit of detection (LOD) were studied and improved. We also present a new coupling approach based on AP IR-MALDI where single droplets generated by a piezoactuated flow-through cell are desorbed and ionized. The potential application of these two coupling methods for LC-MS coupling are discussed.

\section{Experimental}

\section{Instrumentation}

All UV experiments were conducted on a prototype orthogonal-injection TOF mass spectrometer from Agilent (USA), consisting of a differentially pumped interface to the TOF mass analyzer with a heatable transfer capillary, two skimmers, and an octopol ion guide for collisional cooling and focusing. The mass spectrometric interface exhibits a cutoff for masses below $120 \mathrm{Da}$ and above 7,500 Da. Ions are orthogonally extracted by a pulsed extraction field at a repetition rate of $4 \mathrm{kHz}$ and guided via a single-stage reflectron to the detector (Burle, USA). The detector signal is amplified and digitized with a computer (Hewlett Packard, USA) at a sampling frequency of $250 \mathrm{MHz} .10,000$ to 100,000 transients were summed and transferred to a PC for further processing. All mass spectra in this paper are displayed without a $Y$ scale because the intensities are in "arbitrary units."

The homebuilt AP MALDI source is shown in Fig. 1. To obtain an analyte/matrix mixture, the analyte solution was premixed with the matrix solution and infused. In principle, the mixing could be done after separation by a LC column using a mixing tee, as demonstrated previously by Nagra and $\mathrm{Li}$ [24]. The analyte/matrix mixture is fed into the fused silica exit capillary $(50-\mu \mathrm{m}$ inner diameter, $360-\mu \mathrm{m}$ outer diameter, Upchurch Scientific, USA) by a high-pressure LC (HPLC) coupling. The mixture is delivered through this capillary with a syringe pump (Harvard Apparatus, USA) at

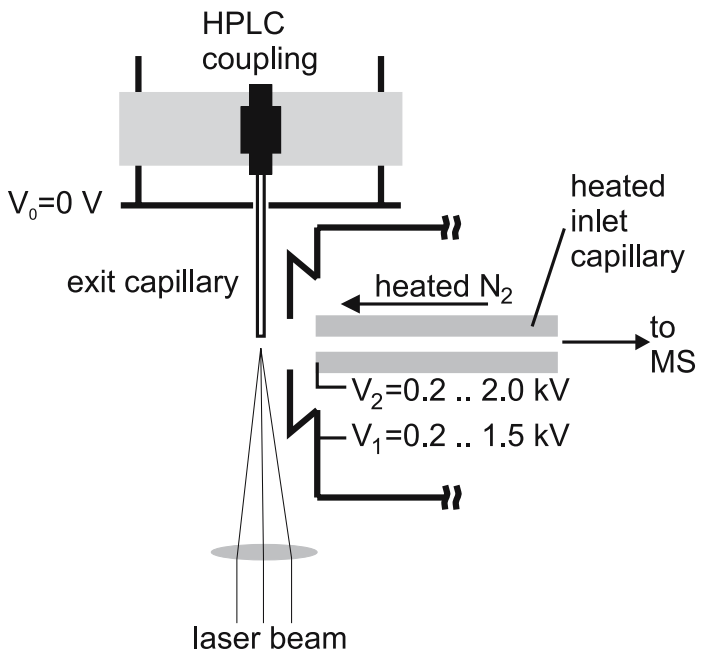

Fig. 1 Flow-injection setup used for atmospheric pressure $(A P)$ UV matrix assisted laser desorption/ionization $(M A L D I)$. The analyte/ matrix mixture is fed into the fused silica exit capillary by a highperformance liquid chromatography (HPLC) coupling. A pulsed laser beam is focused onto the droplets forming at the end of the exit capillary, desorbing and ionizing the matrix and analyte. The ions produced are guided by electric fields to the inlet of the mass spectrometer interface

flow rates between 0.5 and $10 \mu \mathrm{l} / \mathrm{min}$, which are compatible with $\mu$-HPLC and HPLC applications. A small droplet forms at the end of the capillary. A pulsed laser beam is focused from below onto the surface of the droplet, desorbing and ionizing matrix and analytes. The ions produced are guided by electric fields and by a gas flow to the inlet of the mass spectrometer interface. Two different UV lasers were tested to generate the ions: a nitrogen laser $(337 \mathrm{~nm}, 20 \mathrm{~Hz}, 200 \mu \mathrm{J} /$ pulse, Laser Science, USA) and a "Power Chip" laser (Nd:YAG, $355 \mathrm{~nm}, 1 \mathrm{kHz}, 15 \mu \mathrm{J} / \mathrm{pulse}$, JDS Uniphase, USA). The repetition rate laser gives a higher duty cycle for ion production, resulting in higher sensitivity.

All IR experiments were conducted on an ion trap mass analyzer (LCQ Classic QIT, Thermo Finnigan, USA). For "deposited-droplet" measurements a substantially modified AP MALDI source (model 110, MassTech, USA) was used [25]. For single-droplet measurements the AP MALDI source shown in Fig. 2 was used. Droplets were generated by a piezoactuated flow-through cell (Picology, Sweden) and directed towards the mass spectrometer interface. The laser, synchronized to the droplet ejection, was focused perpendicular to the trajectory onto the flying droplet shortly before the inlet to the mass spectrometer. For ionization a powerful Yb:YAG-pumped optical parametric oscillator infrared laser was used (SESI, USA). This laser provided 30 -ns pulses with an energy per pulse of $0.5-0.8 \mathrm{~mJ}$, with a repetition rate of $5 \mathrm{~Hz}$. The wavelength of the laser was tunable in the region $2.8-3.1 \mu \mathrm{m} ; 2.94 \mu \mathrm{m}$ was utilized in this work. In previous AP IR-MALDI studies [10, 20, 26], a high voltage of typically $2-3 \mathrm{kV}$ was applied to the sample target plate to create a potential difference between the 


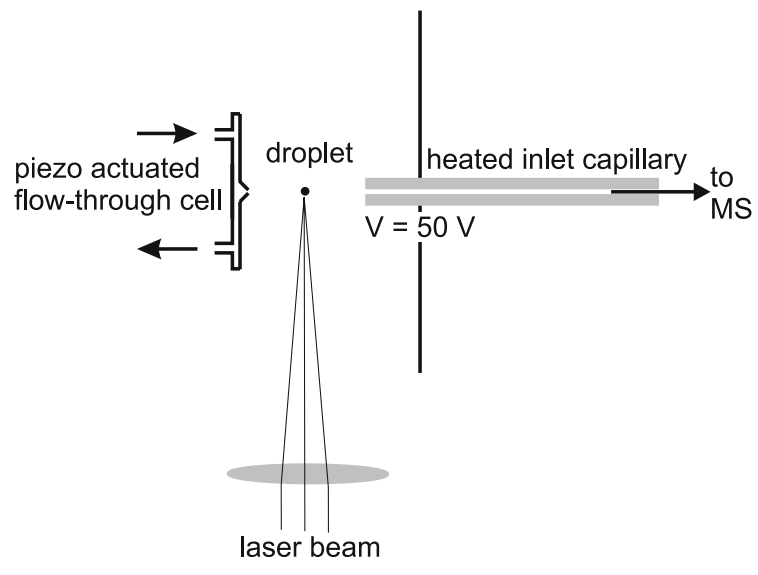

Fig. 2 Setup used for single-droplet IR experiments. The droplets are generated by a piezoactuated flow-through cell. The laser, synchronized to the droplet ejection, is focused onto the flying droplet desorbing the analytes

sample and the intake capillary. However, according to recent findings $[25,27]$ this is not really necessary and therefore no electric field was used in this part of the work.

\section{Materials}

The MALDI matrices $\alpha$-cyano-4-hydroxycinnamic acid (HCCA) and glycerol as well as the additive 3-nitrobenzyl alcohol (NBA) were purchased from Fluka (Switzerland). The peptides angiotensin II, bradykinin, and the antibiotic actinomycin D were obtained from Fluka. The phosphorylated and nonphosphorylated variants of the peptide pp60 c-src (521-533) were purchased from Bachem (Switzerland). The proteins insulin, ubiquitin, and cytochrome $c$ were obtained from Sigma (USA). The oligosaccharides $\alpha$ - , $\beta$, and $\gamma$-cyclodextrin were purchased from Sigma. Molecular-biology-grade water was purchased from Cambrex (USA) and HPLC-grade methanol from Fluka. All solvents, matrices, and analytes were obtained at the highest purity available and were used as received.

\section{Results and discussion}

The AP MALDI interface used in our laboratory [22] lacked sensitivity compared with the signal levels achievable using an ESI source on the same instrument. Therefore, the first goal consisted in optimizing the AP UV-MALDI setup such as to maximize the ion transmission and the sensitivity. The inlet architecture was modified in the following fashion (Fig. 1): (1) a counter current of heated nitrogen was added, and (2) a second counter electrode was introduced. In this new configuration the ions are dragged more efficiently into the interface, not only by means of the electric field, but also by the aerodynamic flow of nitrogen into the inlet. The nitrogen flow, capillary temperature, and the voltages had to be carefully optimized. The LOD is derived from the data in Fig. 3: for a sample flow rate of $0.5 \mu 1 / \mathrm{min}$, a concentration of $0.05 \mu \mathrm{M}$, and an averaging time of $20 \mathrm{~s}$, the amount of sample consumed for this spectrum was $0.5 \times(20 / 60) / 1,000,000 \times 0.05=8.3^{\times} 10^{-15} \mathrm{~mol}=8.3 \mathrm{fmol}$. Compared with our earlier results [22], the LOD could be lowered by at least 10 times, from 83 to $8.3 \mathrm{fmol}$. If a signal-to-noise ratio of 3 is taken as a criterion, the LOD is even lower.

To show the range of applicability of flow-injection AP UV-MALDI, different classes of compounds were measured with HCCA codissolved in methanol. Fig. 4 shows a spectrum of a mixture containing the oligosaccharides $\alpha$-, $\beta$-, and $\gamma$-cyclodextrin. As expected from the structure of the compounds no protonated species can be observed. Rather, sodiated as well as potassiated molecular ion peaks were detected for all three species. For the $\beta$ - and $\gamma$ cyclodextrins the sodiated matrix adducts were also observed.

As proteomics has been evolving in recent years, a major interest concerns the posttranslational modification of proteins and peptides. Two of the most frequent posttranslational modifications are glycosylation and phosphorylation. To determine the site of modification, proteins are usually digested and the fragments are analyzed. Fig. 5a
Fig. 3 Flow-injection AP MALDI of $0.05 \mu \mathrm{M}$ actinomycin $\mathrm{D}, 10 \mu \mathrm{M}$ angiotensin II, $25 \mathrm{mM} \alpha$-cyano-4-hydroxycinnamic acid (HCCA) in methanol. Sample flow rate $0.5 \mu \mathrm{l} /$ min, laser repetition rate $20 \mathrm{~Hz}$

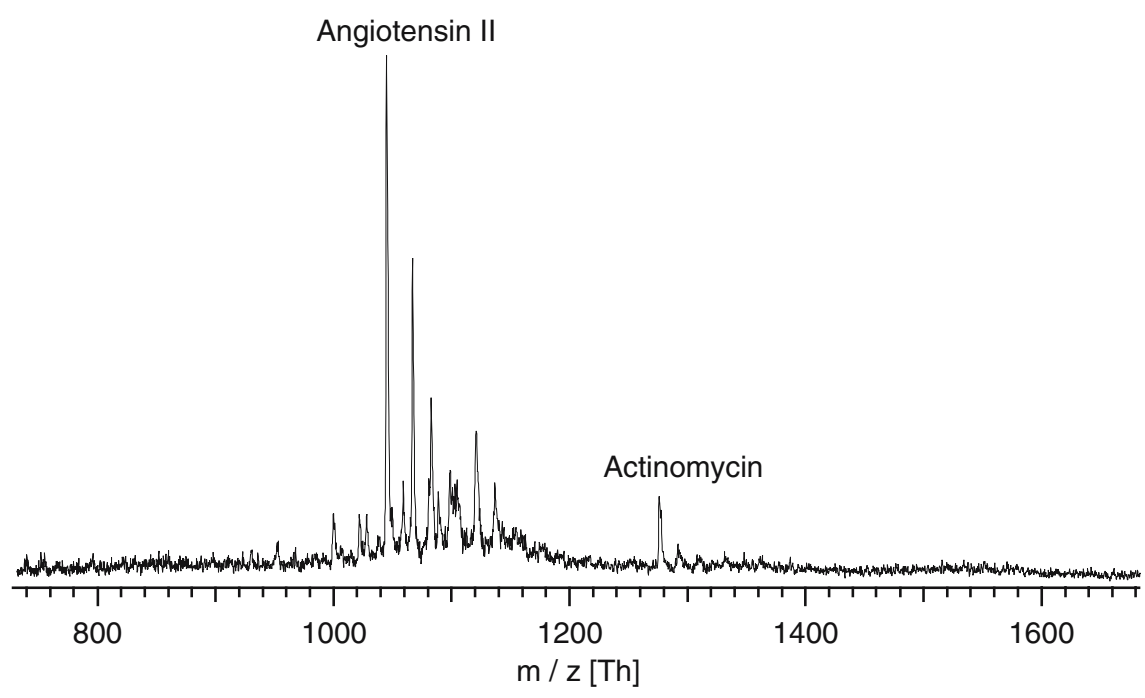


Fig. 4 Flow-injection AP MALDI spectra of $10 \mu \mathrm{M} \alpha$-, $\beta$-, and $\gamma$-cyclodextrins, $25 \mathrm{mM}$ HCCA in methanol pumped with a flow rate of $1 \mu \mathrm{l} / \mathrm{min}$

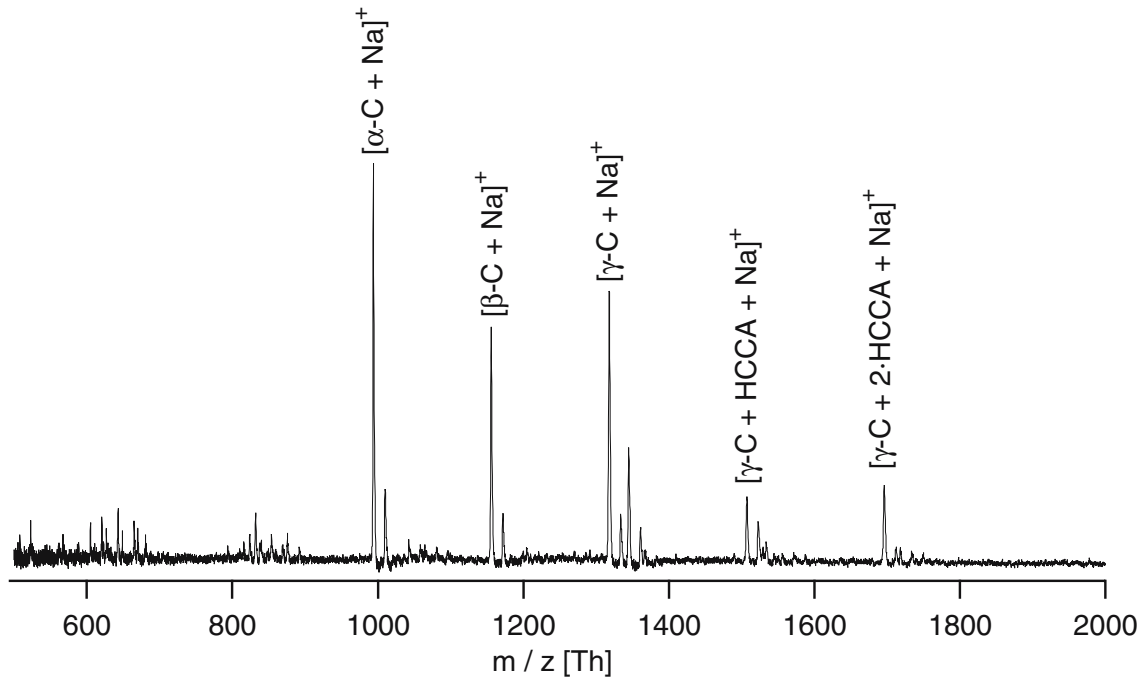

shows the antibiotic actinomycin $\mathrm{D}$ and a mass spectrum obtained from $13 \mu \mathrm{M}$ in methanol with $25 \mathrm{mM}$ HCCA. Owing to the lack of any favorable protonation sites, the sodiated and potassiated molecular ion peaks were again detected with a very good signal-to-noise ratio. Only very minor fragmentation is observed in Fig. 5a. Fig. 5b presents a spectrum of a mixture of pp60 c-src (521-533) in its nonphosphorylated (H3256) and phosphorylated (H3258)
Fig. 5 Flow-injection AP

MALDI spectra of a $13 \mu \mathrm{M}$ actinomycin $\mathrm{D}(A), 25 \mathrm{mM}$ HCCA in methanol pumped with a flow rate of $1 \mu \mathrm{l} / \mathrm{min}$ and b $5 \mu \mathrm{M}$ pp60 c-src (521-533) phosphorylated (H3258) and $5 \mu \mathrm{M}$ pp60 c-src (521-533) nonphosphorylated (H3250), $25 \mathrm{mM}$ HCCA in methanol pumped with a flow rate of $1 \mu \mathrm{l} /$ min. The inset in a shows the structure of actinomycin D. The detector was oversaturated in a, resulting in a slight undershoot

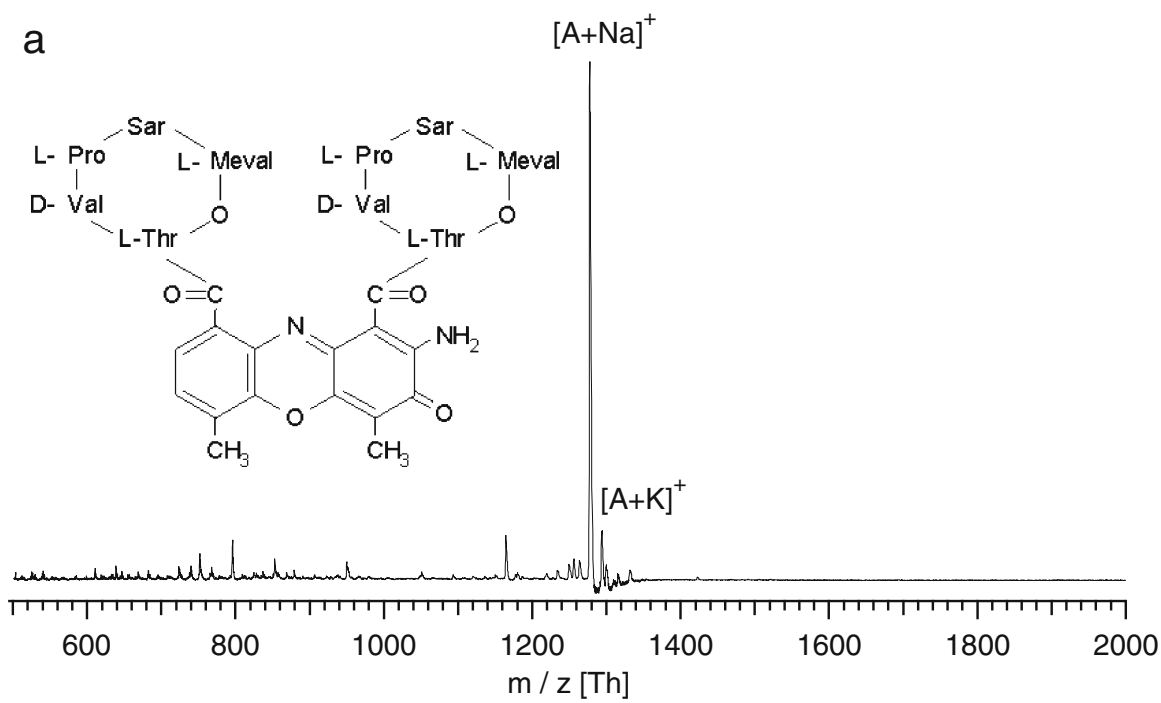

b

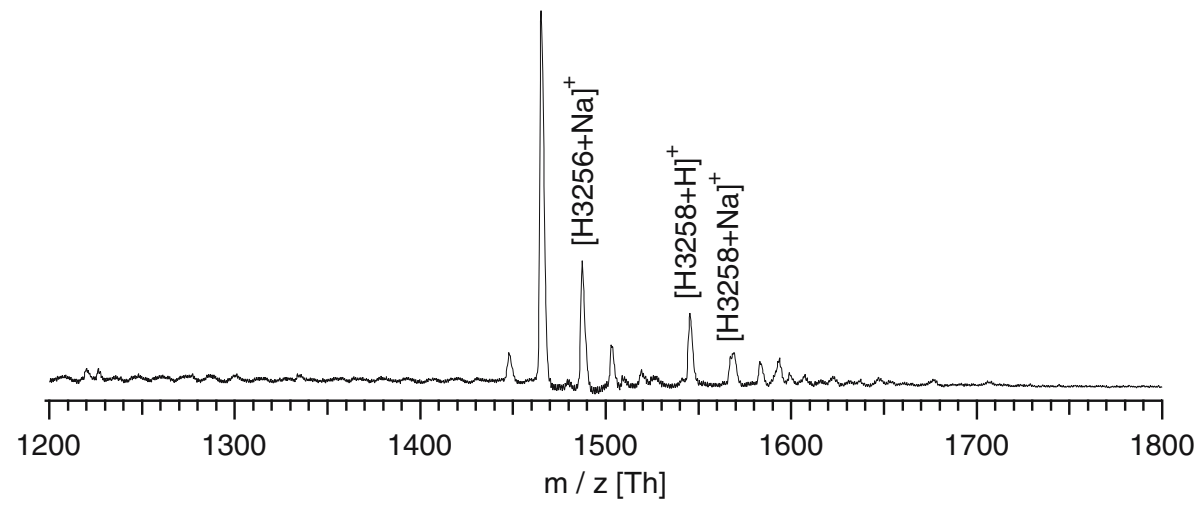


forms, $5 \mu \mathrm{M}$ each with $25 \mathrm{mM}$ HCCA in methanol. For both species the protonated as well as the sodiated and potassiated molecular ion peaks were observed. Although the signal of the nonphosphorylated peptide is about 4 times higher, the phosphorylated peptide shows a fairly good signal intensity as well.

Not only the classes of analytes suitable for analysis by flow-injection AP UV-MALDI are important but also the upper mass limit is of interest. Fig. 6 shows the spectra of ubiquitin $(8,565 \mathrm{Da})$ and cytochrome $c$ (approximately $13,000 \mathrm{Da}$ ). For both ubiquitin and cytochrome $c$, only the doubly and triply charged molecular ions could be observed. This is because the upper $m / z$ limit of the AP interface of the TOF instrument is around 7,500 Th. The low ion yield of the doubly and especially triply charged ions prevented the measurement of higher-mass analytes. Sze et al. [11] showed spectra of bovine serum albumin $(67,000 \mathrm{Da})$ recorded from a liquid matrix consisting of glycerol, HCCA, and 3-aminoquiniline with conventional vacuum MALDI and Ding et al. [28] showed the successful measurement of myoglobin by AP MALDI. We are thus confident that the upper mass limit is not reached yet, that cytochrome $c$ ion at approximately $13,000 \mathrm{Da}$ is produced as expected for MALDI, but is simply not observed owing to the upper mass cutoff of the ion optics. Unfortunately most AP mass spectrometric interfaces, especially on ESI instruments, are optimized for an $\mathrm{m} / \mathrm{z}$ range between 1 and 6,000 Th and do not transmit high-mass ions efficiently, making it difficult or impossible to detect high-mass compounds by AP MALDI. Therefore the application of AP MALDI may currently be limited by instrumental factors to compounds with molecular masses less then 6,000 Da, for example, peptides and small proteins.

For the goal of coupling LC on-line to flow-injection MALDI, it would be beneficial to work without any codissolved matrix, i.e., if the solvent of the separation itself could be employed as a matrix. Especially if separation techniques with low flow rates are employed, mixing two liquid streams with flow rates lower than $1 \mu \mathrm{l} / \mathrm{min}$ homogenously is quite challenging. Therefore the UV laser was exchanged for an IR laser emitting at a wavelength of $2.94 \mu \mathrm{m}(\mathrm{O}-\mathrm{H}$ stretching vibration) with the aim of using water as the matrix. As previously reported by Laiko et al. [20], the addition of small amounts of glycerol should enhance the ion yield for analytes enormously. In order to find the best conditions, an off-line experiment was first carried out. The glycerol content of deposited droplets was increased systematically and the integral of angiotensin II
Fig. 6 Flow-injection AP MALDI of a $15 \mu \mathrm{M}$ ubiquitin, $50 \mathrm{mM}$ HCCA in methanol pumped with a flow rate of $1 \mu \mathrm{l} /$ min and b $10 \mu \mathrm{M}$ cytochrome $c$, $3 \mu \mathrm{M}$ formic acid, $25 \mathrm{mM}$ HCCA in $60 \%$ methanol, $32 \%$ acetonitrile and $8 \%$ water pumped with a flow rate of $1 \mu \mathrm{l} /$ min. Only the doubly and triply charged ions could be detected

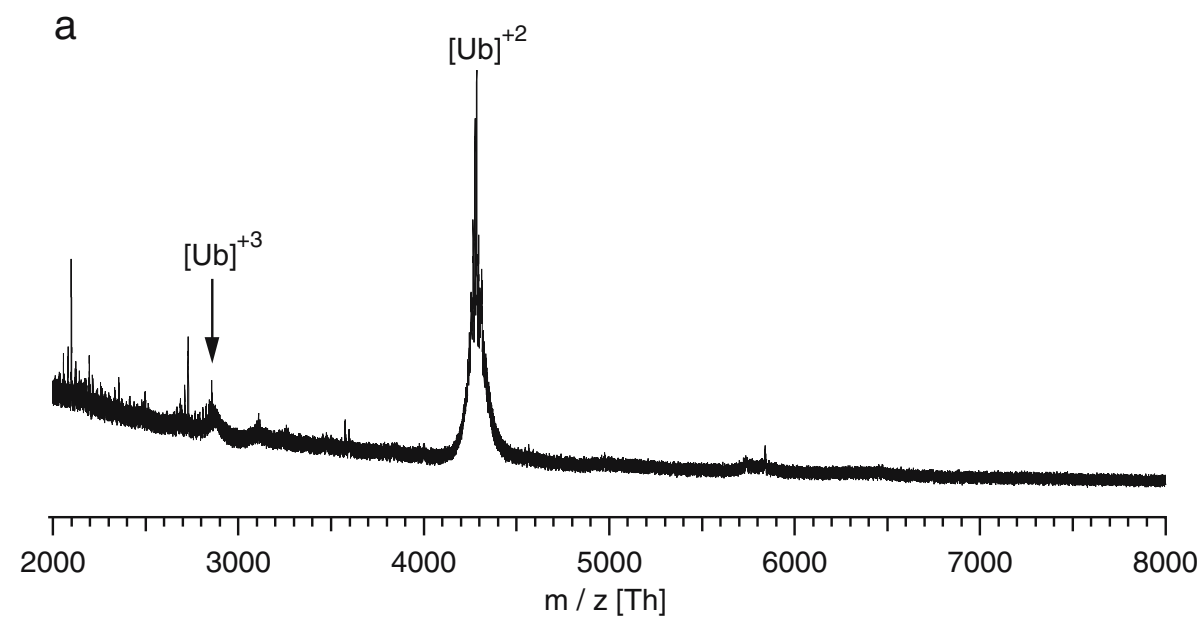

b $[\text { Cyt C] }]^{+2}$

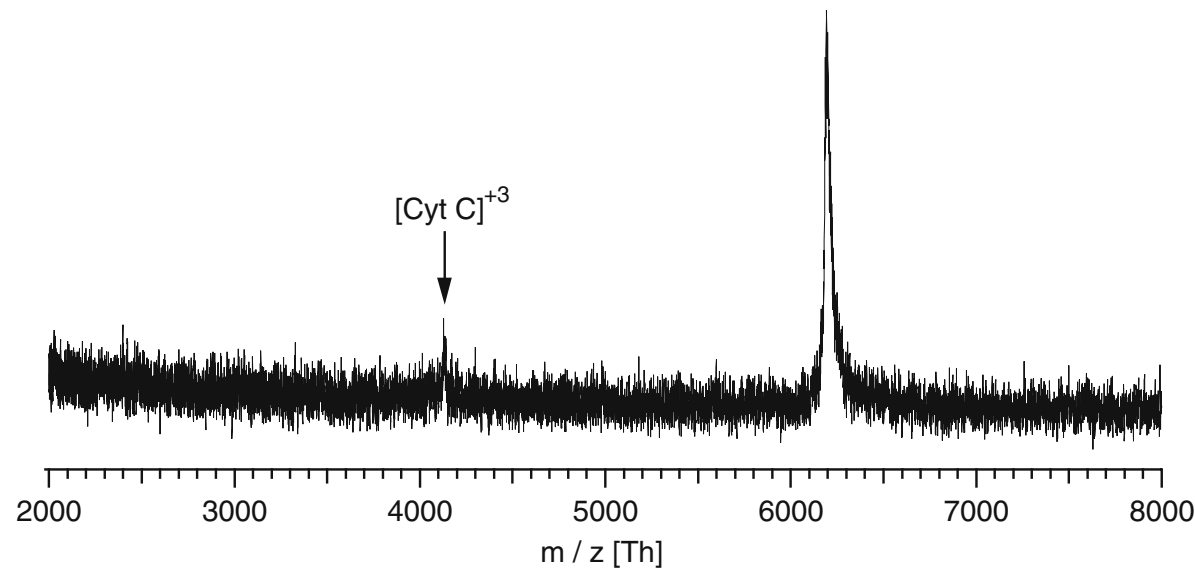


Fig. 7 Signal intensities vs. glycerol content of $5 \mu \mathrm{M}$ angiotensin II in water with 0 60 vol\% glycerol. The spectra were measured off-line by AP IR-MALDI using deposited droplets as samples

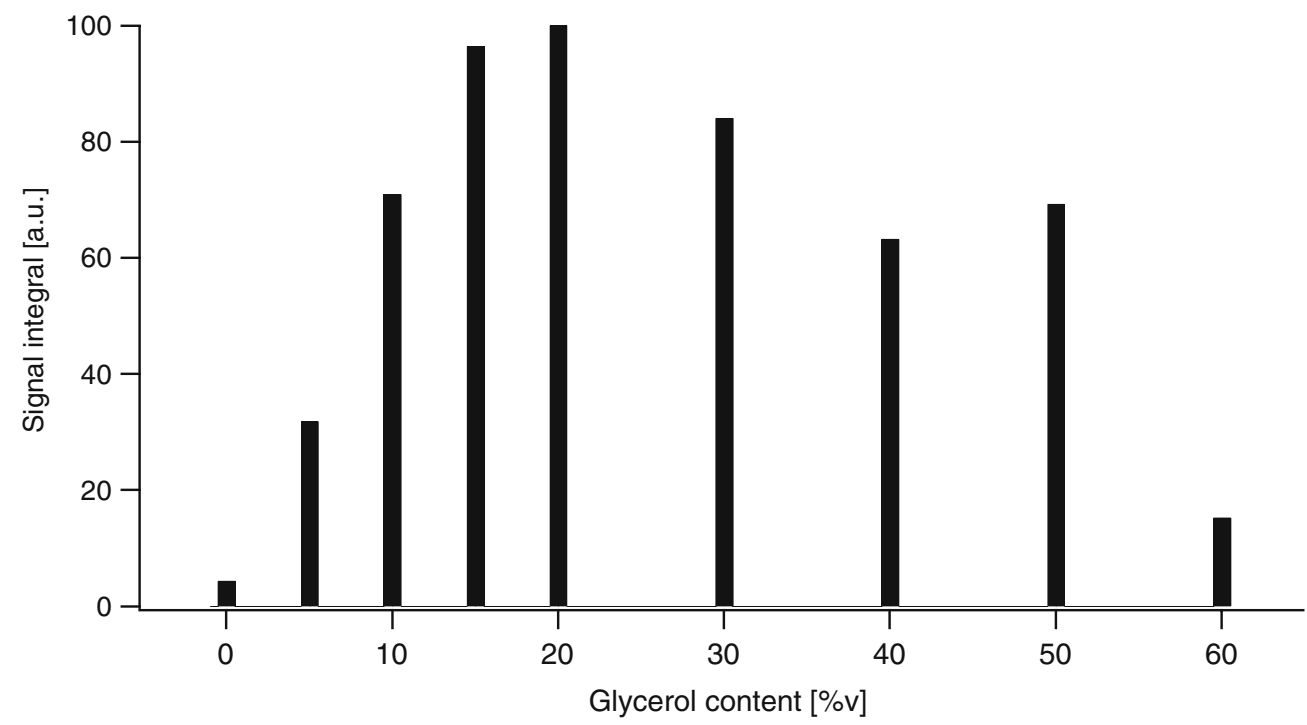

was used to observe the signal response (Fig. 7). This experiment was not done with the piezo valve, but rather by pipetting, i.e., the droplet area was much larger than the laser spot. The best conditions could be found for a glycerol content of $20 \mathrm{vol} \%$, improving the signal-to-noise ratio of angiotensin by a factor of 8 . Interestingly, the addition of $1 \mathrm{vol} \%$ NBA also resulted in a signal improvement, by a factor of 3 (data not shown). Using a mixture of
Fig. 8 Single-droplet AP IRMALDI of a $15 \mu \mathrm{M}$ angiotensin II, $15 \mu \mathrm{M}$ bradykinin in water and b $15 \mu \mathrm{M}$ angiotensin II, $15 \mu \mathrm{M}$ bradykinin, 10 vol\% glycerol in water. The laser repetition rate as well as the droplet generation frequency was $5 \mathrm{~Hz}$. The signals around $1,100 \mathrm{Th}$ in $\mathbf{b}$ are due to cluster formation of analyte and matrix
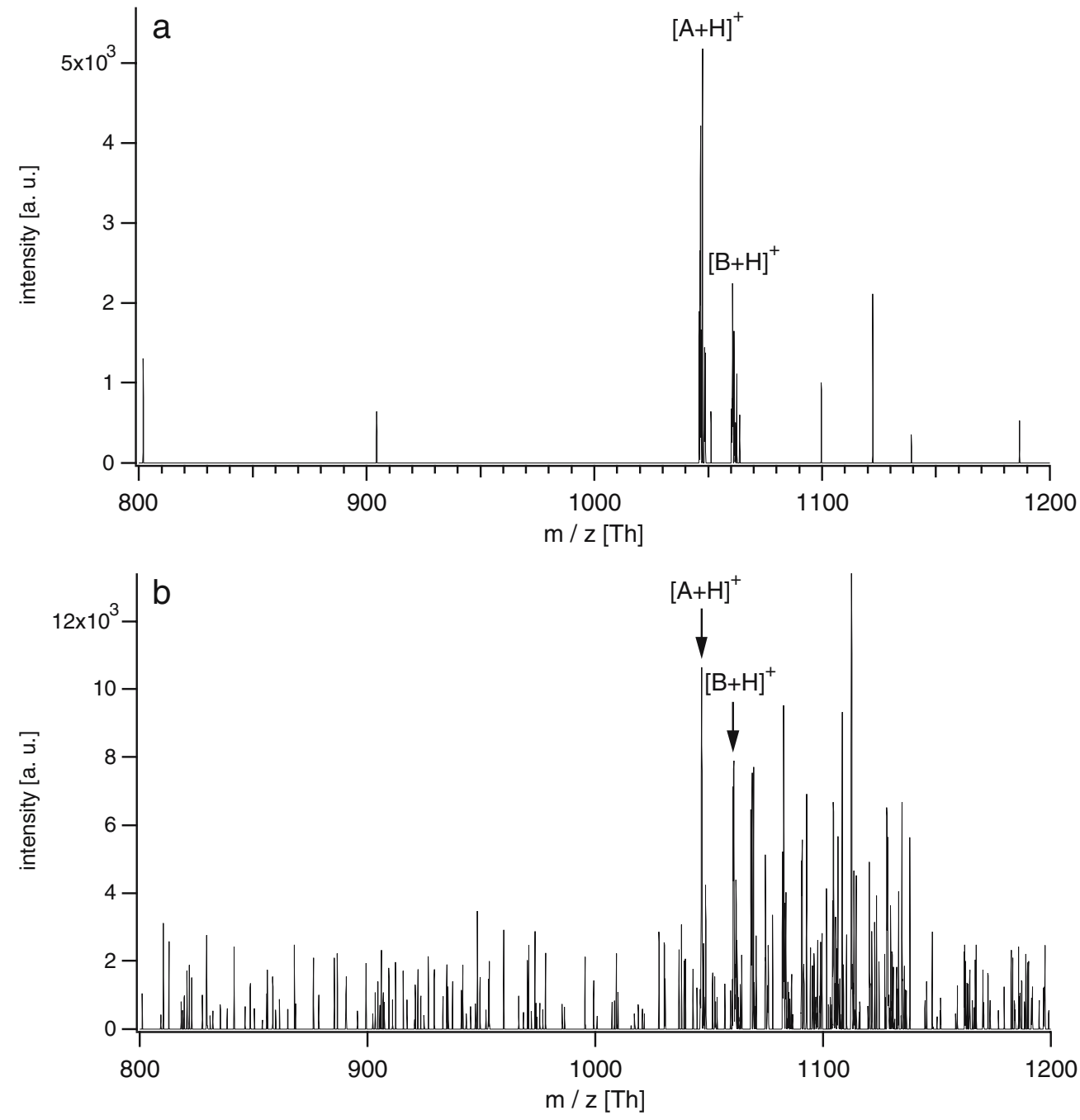
angiotensin II and bradykinin, we observed different relative abundances of the two molecular ion peaks for glycerol or NBA. Actually, even a high intensity of the doubly charged molecular ion signal of bradykinin could be observed using $1 \mathrm{vol} \%$ NBA. This could be interesting for collision-induced dissociation MS/MS experiments, because the confidence level of protein identification is raised using doubly charged ion as parent ions [29]. According to the IR absorption spectra, glycerol absorbs only weakly at $2.94 \mu \mathrm{m}$ and NBA does not absorb at all at this wavelength. This indicates that not only the absorbance of the matrix mixture but also other properties of the sample solution, such as surface tension and viscosity, play an important role for successful desorption and ionization.

The best way to guarantee high reproducibility is when the sample conditions for each desorption/ionization are identical. By using especially designed piezoactuated flowthrough cell this can be achieved [30]. A liquid stream of analyte was fed into the flow-through cell. By applying a pulsed voltage to the piezo element of the cell, almost identical droplets are ejected with a velocity of approximately $1 \mathrm{~m} / \mathrm{s}$ orthogonal to the sample flow, in the direction of the mass spectrometric interface. By carefully synchronizing the droplet ejection and the laser pulse, it was possible to focus the laser onto the flying droplets. Fig. 8 shows a spectrum of $15 \mu \mathrm{M}$ angiotensin II, $15 \mu \mathrm{M}$ bradykinin in water and of $15 \mu \mathrm{M}$ angiotensin II, $15 \mu \mathrm{M}$ bradykinin, $10 \mathrm{vol} \%$ glycerol in water. A weak signal of the molecular ions could be observed and astonishingly little chemical noise was recorded when using pure water as the matrix. Surprisingly, the addition of glycerol did not improve the signal-to-noise ratio. Rather, the opposite was true: much more chemical noise was detected [by comparing the spectra obtained from analyte/water (Fig. 8a) and analyte/water/glycerol (Fig. 8b), it is obvious that the origin of the noise must be linked to the glycerol content, which is the only difference in these samples]. This observation is in contradiction to the results found for deposited droplets. There are three major differences for deposited-droplet and flying-droplet desorption experiments which may account for the different behavior:

1. The shape of the irradiated surface. While the surface of the deposited droplet is approximately flat, the flying droplet's surface is spherical.

2. The deposited droplet interacts with the sample target plate, whereas the flying droplet does not have any interaction with a solid support.

3. The droplet size is dependent on the surface tension and on the viscosity of the solution. It has been demonstrated that the droplet size is smaller for lower surface tension [31], but the behavior for solutions with higher viscosities has not been investigated.

There is clearly need for more research about the exact mechanism and more experiments will be necessary to fully explain this behavior.

According to the manufacturer of the piezoactuated flow-through cell, the diameter of the emitted droplets is $50 \mu \mathrm{m}$ [30]. Using a concentration of $15 \mu \mathrm{M}$ peptide the resulting amount of analyte is as low as 980 amol per droplet; therefore, only a very weak signal is expected. Using a repetition rate of $5 \mathrm{~Hz}$ results in a sample consumption of approximately $500 \mathrm{pl} / \mathrm{min}$; thus, only $0.05 \%$ of a sample delivered with $1 \mu \mathrm{l} / \mathrm{min}$ is consumed. We are confident that the signal-to-noise ratio could be improved by using a higher repetition rate IR laser and an equally high rate of droplet ejection. Unfortunately, high repetition rate IR lasers are not readily available. Nevertheless, we predict that this will become an ideal strategy for coupling to LC, once the sensitivity is improved, as (1) no additional matrix is needed if water is present in the effluent, (2) a high reproducibility is expected, and (3) the system is easily interfaced with common ESI mass spectrometers.

\section{Conclusion}

The wide applicability of liquid AP UV-MALDI has been demonstrated for peptides, actinomycin antibiotics, phosphopeptides, and for oligosaccharides. It was shown that the current upper mass limit of 7,500 Th is due to instrumental parameters rather than to restrictions of the desorption/ionization method. The LOD was found to be 8.3 fmol using dissolved HCCA as a matrix. The main disadvantage of this method related to coupling to LC is the necessity to mix the effluent with a solution of the matrix. Not only the process of mixing can be problematic, but also the solubility of the matrix is dependent on the effluent composition. This has the risk that matrix can precipitate and clog the system. Apart from that, the method offers a good perspective for on-line interfacing: good sensitivity, high salt tolerance, and, as shown in previous work [22], the possibility of quantitation.

The performance of pure water and water containing different amounts of glycerol and NBA as a matrix for AP IR-MALDI was investigated. It was found that for a deposited droplet, water containing $20 \mathrm{vol} \%$ glycerol gives the best results. A single-droplet AP IR-MALDI experiment revealed that for single-droplet desorption/ionization, the addition of glycerol is not beneficial. The overall sensitivity achieved using an IR laser with a repetition rate of $5 \mathrm{~Hz}$ was low. Nevertheless, in our opinion this method shows great potential for coupling to LC if a higher repetition rate IR laser is used. There is no additional mixing step needed prior to the desorption, if the effluents contain a sufficient fraction of water.

Acknowledgements We thank SESI (Columbia, MD, USA) and its team for the loan and support of the IR laser and JDS Uniphase and GMP SA for the loan of the Power Chip laser.

Partial financial support from the Swiss National Science Foundation (grant no. 200020-103616) is gratefully acknowledged.

\section{References}

1. Karas M, Bachmann D, Hillenkamp F (1985) Anal Chem 57: 2935-2939 
2. Karas M, Bachmann D, Bahr U, Hillenkamp F (1987) Int J Mass Spectrom Ion Process 78:53-68

3. Karas M, Hillenkamp F (1988) Anal Chem 60:2299-2301

4. Tanaka K, Waki H, Ido Y, Akita S, Yoshida Y, Yoshida T (1988) Rapid Commun Mass Spectrom 2:151-153

5. Macha SF, Limbach PA, Savickas PJ (2000) J Am Soc Mass Spectrom 11:731-737

6. Przybilla L, Brand JD, Yoshimura K, Rader HJ, Mullen K (2000) Anal Chem 72:4591-4597

7. Armstrong DW, Zhang LK, He LF, Gross ML (2001) Anal Chem 73:3679-3686

8. Lin YS, Chen YC (2002) Anal Chem 74:5793-5798

9. Berkenkamp S, Kirpekar F, Hillenkamp F (1998) Science 281:260-262

10. Von Seggern CE, Moyer SC, Cotter RJ (2003) Anal Chem 75:3212-3218

11. Sze ETP, Chan TWD, Wang G (1998) J Am Soc Mass Spectrom 9:166-174

12. Wei J, Buriak JM, Siuzdak G (1999) Nature 399:243-246

13. Go EP, Prenni JE, Wei J, Jones A, Hall SC, Witkowska HE, Shen ZX, Siuzdak G (2003) Anal Chem 75:2504-2506

14. Laiko VV, Baldwin MA, Burlingame AL (2000) Anal Chem 72:652-657

15. Laiko VV, Moyer SC, Cotter RJ (2000) Anal Chem 72:52395243

16. Doroshenko VM, Laiko VV, Taranenko NI, Berkout VD, Lee HS (2002) Int J Mass Spectrom 221:39-58

17. Wolfender JL, Chu FX, Ball H, Wolfender F, Fainzilber M, Baldwin MA, Burlingame AL (1999) J Mass Spectrom 34:447454
18. Ring S, Rudich Y (2000) Rapid Commun Mass Spectrom 14:515-519

19. Zöllner P, Stubiger G, Schmid E, Pittenauer E, Allmaier G (1997) Int J Mass Spectrom 169:99-109

20. Laiko VV, Taranenko NI, Berkout VD, Yakshin MA, Prasad CR, Lee HS, Doroshenko VM (2002) J Am Soc Mass Spectrom $13: 354-361$

21. Lawson SJ, Murray KK (2002) Rapid Commun Mass Spectrom 16:1248-1250

22. Daniel JM, Ehala S, Friess SD, Zenobi R (2004) Analyst 129:574-578

23. Turney K, Harrison WW (2004) Rapid Commun Mass Spectrom 18:629-635

24. Nagra DS, Li L (1995) J Chromatogr A 711:235-245

25. Tan PV, Taranenko NI, Laiko VV, Yakshin MA, Prasad CR, Doroshenko VM (2004) J Mass Spectrom 39:913-921

26. Von Seggern CE, Zarek PE, Cotter RJ (2003) Anal Chem 75:6523-6530

27. Laiko VV, Tan PV, Taranenko NI, Yakshin MA, Prasad CR, Doroshenko VM (2003) Proceedings of the 51st ASMS conference on mass spectrometry and allied topics, Montreal

28. Ding L, Sudakov M, Brancia FL, Giles R, Kumashiro S (2004) J Mass Spectrom 39:471-484

29. Cramer R, Corless S (2001) Rapid Commun Mass Spectrom 22:2058-2066

30. Laurell T, Wallman L, Nilsson J (1999) J Micromech Microeng 9:369-376

31. Önnerfjord P, Nilsson J Wallman L, Laurell T, Marko-Varga G (1998) Anal Chem 70:4755-4760 